\title{
New Non-Symmetric Orthogonal Basis for the Calogero Model with Distinguishable Particles
}

\author{
Hideaki Ujinot, Akinori Nishino and Miki Wadati \\ Department of Physics, Graduate School of Science, University of Tokyo, \\ Hongo 7-3-1, Bunkyo-ku, Tokyo 113-0033, Japan

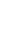

\begin{abstract}
We demonstrate an algebraic construction of all the simultaneous eigenfunctions of the conserved operators for distinguishable particles governed by the Calogero Hamiltonian. Our construction is completely parallel to the construction of the Fock space for decoupled quantum harmonic oscillators. The simultaneous eigenfunction does not coincide with the non-symmetric Hi-Jack polynomial, which shows that the conserved operators derived from the number operators of the decoupled quantum harmonic oscillators are algebraically different from the known ones derived by the Dunkl operator formulation. 03.65.Ge, 03.65.Fd, 02.90.+p
\end{abstract}

Typeset using REVTEX 
There has been a surge of interest in the orthogonal symmetric polynomials associated with the quantum integrable systems with inverse-square long-range interactions. Particularly after the Jack polynomials, which span the orthogonal basis for the Sutherland model [1 4 , enabled an exact calculation of dynamical density-density correlation functions of the model [5], the Jack polynomial and its variants have been extensively studied. So far, we have been studying the Hi-Jack (or multivariable Hermite) symmetric polynomial that forms the symmetric orthogonal basis for the Calogero model [6 10],

$$
\hat{H}_{\mathrm{C}}=\frac{1}{2} \sum_{j=1}^{N}\left(p_{j}^{2}+\omega^{2} x_{j}^{2}\right)+\frac{1}{2} \sum_{\substack{j, k=1 \\ j \neq k}}^{N} \frac{a^{2}-a K_{j k}}{\left(x_{j}-x_{k}\right)^{2}},
$$

where $p_{j}=-\mathrm{i} \frac{\partial}{\partial x_{j}}$. The operator $K_{j k}$ is a coordinate exchange operator that is defined by the action on a multivariable function, $\left(K_{j k} f\right)\left(\cdots, x_{j}, \cdots, x_{k}, \cdots\right)=f\left(\cdots, x_{k}, \cdots, x_{j}, \cdots\right)$. For the symmetric case, the exchange operators at the rightmost of the expressions are identified with the identity, $K_{j k} \equiv 1$. Using the Dunkl operator formulation [11, [2], we derived the Rodrigues formula for the Hi-Jack symmetric polynomial for the first time [8]. The Hi-Jack symmetric polynomials are the simultaneous eigenfunctions of all the commuting conserved operators of the Calogero model derived by the Dunkl operator formulation [9, [10]. They describe the indistinguishable particles that obey the Calogero Hamiltonian and are considered to be multivariable Hermite polynomials with one additional parameter [9]. The name HiJack comes from the fact that the symmetric polynomial is a one-parameter deformation of the Jack polynomial. Sogo showed a transformation of the symmetric Calogero Hamiltonian into the Euler operator $\mathcal{O}_{\mathrm{E}} \stackrel{\text { def }}{=} \sum_{j=1}^{N} x_{j} \frac{\partial}{\partial x_{j}}$ [13]. Recently, Gurappa and Panigrahi showed a transformation of the symmetric Calogero Hamiltonian into decoupled quantum harmonic oscillators [14]. Motivated by their results, we presented an algebraic construction of a new symmetric orthogonal basis for the Calogero Hamiltonian [15] in a completely parallel way to the construction of the Fock space of the bosonic quantum harmonic oscillators. Since the first quantized Hamiltonian itself has no information on the statistics of the particles, there should be eigenfunctions not only for indistinguishable particles but also for distinguishable particles. Namely, there should be non-symmetric eigenfunctions that describe 
distinguishable particles governed by the Calogero Hamiltonian (1). In this letter, we shall show a simple algebraic formula for all the simultaneous eigenfunctions of the conserved operators of the Calogero model with distinguishable particles and discuss the meaning of the transformation into the interaction-free system.

First, we decompose the Calogero Hamiltonian (1) into interaction-free $N$-particle Hamiltonian. The ground state wave function of the Calogero model is given by the real Laughlin wave function,

$$
\phi_{\mathrm{g}}(\boldsymbol{x})=\prod_{1 \leq j<k \leq N}\left|x_{j}-x_{k}\right|^{a} \exp \left(-\frac{1}{2} \omega \sum_{j=1}^{N} x_{j}^{2}\right),
$$

and the ground state energy is given by $E_{\mathrm{g}}=\frac{1}{2} N \omega(N a+(1-a))$. Then the following similarity transformation removes the action to the ground state wave function from the Hamiltonian,

$$
\phi_{\mathrm{g}}^{-1}\left(\hat{H}_{\mathrm{C}}-E_{\mathrm{g}}\right) \phi_{\mathrm{g}}=\omega \mathcal{O}_{\mathrm{E}}-\frac{1}{2} \mathcal{O}_{\mathrm{L}}
$$

where the non-symmetric Lassalle operator $\mathcal{O}_{\mathrm{L}}$ is [16]

$$
\begin{aligned}
\mathcal{O}_{\mathrm{L}}= & \sum_{j=1}^{N} \frac{\partial^{2}}{\partial x_{j}^{2}}+a \sum_{\substack{j, k=1 \\
j \neq k}}^{N}\left(\frac{1}{x_{j}-x_{k}}\left(\frac{\partial}{\partial x_{j}}-\frac{\partial}{\partial x_{k}}\right)\right. \\
& \left.+\frac{K_{j k}-1}{\left(x_{j}-x_{k}\right)^{2}}\right) .
\end{aligned}
$$

As is similar to Sogo's approach to the symmetric case, the Calogero Hamiltonian can be transformed to the Euler operator. The commutation relation $\left[\mathcal{O}_{\mathrm{L}}, \mathcal{O}_{\mathrm{E}}\right]=2 \mathcal{O}_{\mathrm{L}}$ and the Baker-Hausdorff formula yield

$$
\mathrm{e}^{\frac{1}{4 \omega} \mathcal{O}_{\mathrm{L}}}\left(\omega \mathcal{O}_{\mathrm{E}}-\frac{1}{2} \mathcal{O}_{\mathrm{L}}\right) \mathrm{e}^{-\frac{1}{4 \omega} \mathcal{O}_{\mathrm{L}}}=\omega \mathcal{O}_{\mathrm{E}}
$$

In a similar way, we can transform the Euler operator into the Hamiltonian of the decoupled quantum harmonic oscillators. The commutator between the Euler operator $\mathcal{O}_{\mathrm{E}}$ and the Laplacian $\nabla^{2} \stackrel{\text { def }}{=} \sum_{j=1}^{N} \frac{\partial^{2}}{\partial x_{j}^{2}},\left[\nabla^{2}, \mathcal{O}_{\mathrm{E}}\right]=2 \nabla^{2}$, and again the Baker-Hausdorff formula yield

$$
\mathrm{e}^{-\frac{1}{4 \omega} \nabla^{2}} \omega \mathcal{O}_{\mathrm{E}} \mathrm{e}^{\frac{1}{4 \omega} \nabla^{2}}=\omega\left(\mathcal{O}_{\mathrm{E}}-\frac{1}{2 \omega} \nabla^{2}\right)
$$


Another similarity transformation using the Gaussian kernel produces the Hamiltonian for the decoupled $N$ quantum harmonic oscillators,

$$
\mathrm{e}^{-\frac{1}{2} \omega \boldsymbol{x}^{2}} \omega\left(\mathcal{O}_{\mathrm{E}}-\frac{1}{2 \omega} \nabla^{2}\right) \mathrm{e}^{\frac{1}{2} \omega \boldsymbol{x}^{2}}=\frac{1}{2}\left(\sum_{j=1}^{N}\left(p_{j}^{2}+\omega^{2} x_{j}^{2}\right)-N \omega\right),
$$

where the abbreviation $\boldsymbol{x}^{2} \stackrel{\text { def }}{=} \sum_{j=1}^{N} x_{j}^{2}$ is used. In terms of the number $n_{j} \stackrel{\text { def }}{=} a_{j}^{\dagger} a_{j}$ creation $a_{j}^{\dagger} \stackrel{\text { def }}{=} \frac{1}{2 \mathrm{i} \omega}\left(p_{j}+\mathrm{i} \omega x_{j}\right)$ and annihilation $a_{j} \stackrel{\text { def }}{=} \mathrm{i}\left(p_{j}-\mathrm{i} \omega x_{j}\right)$ operators, the above relation is expressed by

$$
\mathrm{e}^{-\frac{1}{2} \omega \boldsymbol{x}^{2}} \omega\left(\mathcal{O}_{\mathrm{E}}-\frac{1}{2 \omega} \nabla^{2}\right) \mathrm{e}^{\frac{1}{2} \omega \boldsymbol{x}^{2}}=\omega \sum_{j=1}^{N} n_{j} .
$$

In summary, successive applications of the similarity transformations transform the Calogero Hamiltonian $\hat{H}_{\mathrm{C}}$ (1) to the Hamiltonian of the decoupled $N$ quantum harmonic oscillators.

Since the number operators, $n_{j}, j=1, \cdots, N$, mutually commute, these conserved operators are simultaneously diagonalizable. Thus the non-degenerate simultaneous eigenfunctions of the number operators, which are nothing but the number states, form the non-symmetric orthogonal basis of the Hilbert (Fock) space for the $N$ decoupled harmonic oscillators. The similarity transformation indicates that the non-symmetric orthogonal basis for the Calogero model can be constructed exactly in the same manner. Defining the creation, annihilation and number operators by

$$
\begin{aligned}
& \hat{b}_{j}^{+} \stackrel{\text { def }}{=} \phi_{\mathrm{g}} \mathrm{e}^{-\frac{1}{4 \omega} \mathcal{O}_{\mathrm{L}}} \mathrm{e}^{\frac{1}{4 \omega} \nabla^{2}} \mathrm{e}^{\frac{1}{2} \omega \boldsymbol{x}^{2}} a_{j}^{\dagger} \mathrm{e}^{-\frac{1}{2} \omega \boldsymbol{x}^{2}} \mathrm{e}^{-\frac{1}{4 \omega} \nabla^{2}} \mathrm{e}^{\frac{1}{4 \omega} \mathcal{O}_{\mathrm{L}}} \phi_{\mathrm{g}}^{-1}, \\
& \hat{b}_{j} \stackrel{\text { def }}{=} \phi_{\mathrm{g}} \mathrm{e}^{-\frac{1}{4 \omega} \mathcal{O}_{\mathrm{L}}} \mathrm{e}^{\frac{1}{4 \omega} \nabla^{2}} \mathrm{e}^{\frac{1}{2} \omega \boldsymbol{x}^{2}} a_{j} \mathrm{e}^{-\frac{1}{2} \omega \boldsymbol{x}^{2}} \mathrm{e}^{-\frac{1}{4 \omega} \nabla^{2}} \mathrm{e}^{\frac{1}{4 \omega} \mathcal{O}_{\mathrm{L}}} \phi_{\mathrm{g}}^{-1}, \\
& \hat{n}_{j}^{\mathrm{C}} \stackrel{\text { def }}{=} \hat{b}_{j}^{\dagger} \hat{b}_{j},
\end{aligned}
$$

we can algebraically construct the non-symmetric simultaneous eigenfunctions of all the number operators $\hat{n}_{j}^{\mathrm{C}}$ as

$$
\left|\lambda_{\sigma}\right\rangle=\prod_{j=1}^{N}\left(\hat{b}_{j}^{+}\right)^{\lambda_{\sigma(j)}}|0\rangle \stackrel{\text { def }}{=} M_{\lambda_{\sigma}}(\boldsymbol{x} ; 1 / a, \omega) \phi_{\mathrm{g}}, \quad|0\rangle \stackrel{\text { def }}{=} \phi_{\mathrm{g}},
$$

where $\lambda$ and $\sigma$ are respectively the Young diagram, $\lambda \stackrel{\text { def }}{=}\left\{\lambda_{1} \geq \lambda_{2} \geq \cdots \geq \lambda_{N} \geq 0\right\}$, where $\lambda_{k}$, $k=1, \cdots, N$, are integers, and the permutation, $\sigma \in S_{N}$. The function $M_{\lambda_{\sigma}}(\boldsymbol{x} ; 1 / a, \omega)$ is a 
non-symmetric polynomial. These non-symmetric functions are apparently non-degenerate simultaneous eigenfunctions of the commuting number operators $\hat{n}_{j}^{\mathrm{C}}$. Defining the bras in exactly the same way as we do for the harmonic oscillators,

$$
\left\langle\lambda_{\sigma}\right|=\langle 0| \prod_{j=1}^{N}\left(\hat{b}_{j}\right)^{\lambda_{\sigma(j)}}, \quad\langle 0| \stackrel{\text { def }}{=} \phi_{\mathrm{g}},
$$

which is nothing but the dual of the above kets, we can readily confirm the bras and the kets form the orthogonal basis,

$$
\left\langle\lambda_{\sigma} \mid \mu_{\tau}\right\rangle=\delta_{\lambda_{\sigma}, \mu_{\tau}} \prod_{j=1}^{N} \lambda_{j} !\langle 0 \mid 0\rangle .
$$

See Ref. 16,17 for the vacuum normalization. Thus we have constructed the non-symmetric orthogonal basis, or the Fock space, for the Calogero model with distinguishable particles.

In order to compare the above basis with the known basis given by the Hi-Jack polynomials [8 10], we rewrite the creation-annihilation operators as

$$
\begin{aligned}
& x_{j}=\mathrm{e}^{\frac{1}{4 \omega} \nabla^{2}} \mathrm{e}^{\frac{1}{2} \omega \boldsymbol{x}^{2}} a_{j}^{\dagger} \mathrm{e}^{-\frac{1}{2} \omega \boldsymbol{x}^{2}} \mathrm{e}^{-\frac{1}{4 \omega} \nabla^{2}}, \\
& \frac{\partial}{\partial x_{j}}=\mathrm{e}^{\frac{1}{4 \omega} \nabla^{2}} \mathrm{e}^{\frac{1}{2} \omega \boldsymbol{x}^{2}} a_{j} \mathrm{e}^{-\frac{1}{2} \omega \boldsymbol{x}^{2}} \mathrm{e}^{-\frac{1}{4 \omega} \nabla^{2}} .
\end{aligned}
$$

Then the polynomial parts of the kets (2) is rewritten as

$$
M_{\lambda_{\sigma}}(\boldsymbol{x} ; 1 / a, \omega)=\mathrm{e}^{-\frac{1}{4 \omega} \mathcal{O}_{\mathrm{L}}} \boldsymbol{x}^{\lambda_{\sigma}}
$$

where $\boldsymbol{x}^{\lambda_{\sigma}} \stackrel{\text { def }}{=} x_{1}^{\lambda_{\sigma(1)}} \cdots x_{N}^{\lambda_{\sigma(N)}}$. A comment might be in order. In order to avoid the essential singularity, we have to restrict the operand of the symmetric Lassalle operator to the symmetric functions [15]. On the other hand, action of the non-symmetric Lassalle operator on a monomial yields a polynomial with no essential singularity. As was shown by Baker and Forrester [16], the non-symmetric Hi-Jack polynomials [16, 18, 19 is given by

$$
j_{\lambda_{\sigma}}(\boldsymbol{x} ; 1 / a, \omega)=\mathrm{e}^{-\frac{1}{4 \omega} \mathcal{O}_{\mathrm{L}}} J_{\lambda_{\sigma}}(\boldsymbol{x} ; 1 / a),
$$

where $J_{\lambda_{\sigma}}(\boldsymbol{x} ; 1 / a)$ is the non-symmetric Jack polynomial [20,21]. The non-symmetric Jack polynomials have triangular expansion forms with respect to the monomials, 


$$
J_{\lambda_{\sigma}}(\boldsymbol{x} ; 1 / a)=\boldsymbol{x}^{\lambda_{\sigma}}+\sum_{\mu_{\tau}<\lambda_{\sigma}} v_{\lambda_{\sigma}, \mu_{\tau}}(a) \boldsymbol{x}^{\mu_{\tau}} \stackrel{\text { def }}{=} T_{\lambda_{\sigma}, \mu_{\tau}} \boldsymbol{x}^{\mu_{\tau}}
$$

The order among the pairs of the Young diagrams and the permutations $\lambda_{\sigma}$ are defined by

$$
\mu_{\tau}<\lambda_{\sigma} \Leftrightarrow\left\{\begin{aligned}
&1) \mu \stackrel{\mathrm{D}}{<} \lambda \\
&2) \text { when } \mu=\lambda \text { then the first } \\
& \text { non-vanishing difference } \\
& \tau(i)-\sigma(i)<0
\end{aligned}\right.
$$

where the dominance order $\stackrel{\mathrm{D}}{<}$ is given as

$$
\mu \stackrel{\mathrm{D}}{<} \lambda \Leftrightarrow \mu \neq \lambda,|\mu|=|\lambda| \text { and } \sum_{k=1}^{l} \mu_{k} \leq \sum_{k=1}^{l} \lambda_{k},
$$

for all $l=1, \cdots, N$. We have used here the symbol for the weight of the Young diagram, $|\lambda| \stackrel{\text { def }}{=} \sum_{j=1}^{N} \lambda_{j}$. Equations (4) - (6) show that the non-symmetric Hi-Jack polynomials and the new non-symmetric orthogonal basis are different, though the latter also can be regarded as a multivariable generalization of the Hermite polynomial with one additional parameter. From the above expansion (6), we readily confirm that the non-symmetric Jack polynomials are not the simultaneous eigenfunctions of the operators $x_{j} \frac{\partial}{\partial x_{j}}, j=1, \cdots, N$, and hence the nonsymmetric Hi-Jack polynomials are not the simultaneous eigenfunctions of the commuting number operators $\hat{n}_{j}^{\mathrm{C}}, j=1, \cdots, N$.

It seems rather strange at first sight that the two orthogonal bases are related by a not unitary but triangular matrix $T_{\lambda_{\sigma}, \mu_{\tau}}$. It reflects the fact that the commuting number operators $\hat{n}_{j}^{\mathrm{C}}$ are not Hermitian but self-dual with respect to the exchange of the creation and annihilation operators $\hat{b}_{j}^{+} \leftrightarrow \hat{b}_{j}, j=1, \cdots, N$. That is why the bra $\left\langle\lambda_{\sigma}\right|$ is not given by the corresponding ket itself, but should be identified with a "rotation" of the ket, $\sum_{\mu_{\tau}, \nu_{v}} T_{\lambda_{\sigma}, \mu_{\tau}} T_{\mu_{\tau}, \nu_{v}}\left|\nu_{v}\right\rangle$ in the inner product. On the other hand, the Dunkl operator formulation [8] 12] yields the Hermitian commuting conserved operators for the Calogero model. The Dunkl operators for the Calogero model are listed as

$$
\hat{\alpha}_{l}^{\dagger} \stackrel{\text { def }}{=}-\frac{\mathrm{i}}{2 \omega}\left(p_{l}+\mathrm{i} a \sum_{\substack{k=1 \\ k \neq l}}^{N} \frac{1}{x_{l}-x_{k}} K_{l k}+\mathrm{i} \omega x_{l}\right),
$$




$$
\begin{aligned}
& \hat{\alpha}_{l} \stackrel{\text { def }}{=} \mathrm{i}\left(p_{l}+\mathrm{i} a \sum_{\substack{k=1 \\
k \neq l}}^{N} \frac{1}{x_{l}-x_{k}} K_{l k}-\mathrm{i} \omega x_{l}\right), \\
& \hat{d}_{l} \stackrel{\text { def }}{=} \hat{\alpha}_{l}^{\dagger} \hat{\alpha}_{l}+a \sum_{j=1}^{l-1}\left(K_{j l}-1\right), \quad\left[\hat{d}_{l}, \hat{d}_{m}\right]=0 .
\end{aligned}
$$

In terms of the Dunkl operators, the Hamiltonian for the Calogero model (1) is given by $\hat{H}_{\mathrm{C}}-E_{\mathrm{g}}=\omega \hat{I}_{1}$, where $\hat{I}_{n} \stackrel{\text { def }}{=} \sum_{l=1}^{N}\left(\hat{d}_{l}\right)^{n}, \quad n=1, \cdots, N$. And the Hermitian commuting conserved operators are given by the $\hat{d}_{l}$-operators (and also $\hat{I}_{n}$ ). The polynomial parts of the non-symmetric simultaneous eigenfunctions for all the above commuting Dunkl operators $\hat{d}_{l}$, or equivalently, the conserved operators $\hat{I}_{n}$, are uniquely identified as the non-symmetric Hi-Jack polynomials, which give an orthogonal basis of the Calogero model with respect to the conventional Hermitian inner product. The non-symmetric simultaneous eigenfunctions (2) are not the simultaneous eigenfunctions for these commuting conserved operators.

From the discussions above, we conclude that the Calogero Hamiltonian has two sets of commuting conserved operators which are algebraically inequivalent to each other. We also conclude that two different conserved operators respectively picked up from the two different sets do not commute $\left[\hat{n}_{j}^{\mathrm{C}}, \hat{I}_{k}\right] \neq 0$, for $k \neq 1$, or equivalently, $\left[\hat{n}_{j}^{\mathrm{C}}, \hat{d}_{k}\right] \neq 0$. The Hilbert space of the Calogero Hamiltonian also has two different orthogonal bases that respectively correspond to the simultaneous eigenfunctions for the two sets of commuting conserved operators. This peculiar fact must be due to the large degeneracy of the eigenvalue of the Calogero Hamiltonian (11),

$$
\hat{H}_{\mathrm{C}}\left|\lambda_{\sigma}\right\rangle=\left(\omega|\lambda|+E_{\mathrm{g}}\right)\left|\lambda_{\sigma}\right\rangle
$$

For a particular eigenvalue, say $\omega n+E_{\mathrm{g}}$, the degeneracy is given by the number of pairs of Young diagrams and permutations $\lambda_{\sigma}$ such that $|\lambda|=n$. Existence of two inequivalent sets of conserved operators and two different simultaneous eigenfunctions implies some hidden dynamical symmetry of the Calogero model, as is the case with the hydrogen atom that has the $O(4)$ dynamical symmetry related to the angular momentum and the Runge-Lenz-Pauli vector [22]. 
We should note that our discussions so far on the $\left(A_{N-1^{-}}\right)$Calogero model also holds for the $B_{N^{-}}$Calogero model [23,

$$
\begin{aligned}
\hat{H}_{B_{N}}= & \frac{1}{2} \sum_{j=1}^{N}\left(p_{j}^{2}+\omega^{2} x_{j}^{2}\right)+\frac{1}{2} \sum_{j=1}^{N} \frac{\left(b^{2}-b t_{j}\right)}{x_{j}^{2}} \\
& +\frac{1}{2} \sum_{\substack{j, k=1 \\
j \neq k}}^{N}\left(\frac{a^{2}-a K_{j k}}{\left(x_{j}-x_{k}\right)^{2}}+\frac{a^{2}-a t_{j} t_{k} K_{j k}}{\left(x_{j}+x_{k}\right)^{2}}\right) .
\end{aligned}
$$

where the reflection operator $t_{j}$ is defined by $\left(t_{j} f\right)\left(\cdots, x_{j}, \cdots\right)=f\left(\cdots,-x_{j}, \cdots\right)$. As is similar to the way of Gurappa and Panigrahi for the symmetric case [24], we can decompose the above Hamiltonian into that of the decoupled quantum particles. By the following similarity transformation, we have

$$
\phi_{\mathrm{g}}^{-1}\left(\hat{H}_{B_{N}}-E_{\mathrm{g}}^{B_{N}}\right) \phi_{\mathrm{g}}=\omega \mathcal{O}_{\mathrm{E}}-\frac{1}{2} \mathcal{O}_{\mathrm{L}}^{B_{N}}
$$

where the ground state, the ground state energy and the non-symmetric $B_{N^{-}}$Lassalle operator are [16]

$$
\begin{aligned}
\phi_{\mathrm{g}}(\boldsymbol{x}) & =\prod_{1 \leq j<k \leq N}\left|x_{j}^{2}-x_{k}^{2}\right|^{a} \prod_{j=1}^{N}\left|x_{j}\right|^{b} \exp \left(-\frac{1}{2} \omega \sum_{j=1}^{N} x_{j}^{2}\right), \\
E_{\mathrm{g}}^{B_{N}} & =\frac{1}{2} N \omega(1+2 a(N-1)+2 b), \\
\mathcal{O}_{L}^{B_{N}} & =\sum_{j=1}^{N}\left(\frac{\partial^{2}}{\partial x_{j}^{2}}+\frac{2 b}{x_{j}} \frac{\partial}{\partial x_{j}}+\frac{b}{x_{j}^{2}}\left(t_{j}-1\right)\right) \\
& +a \sum_{\substack{j, k=1 \\
j \neq k}}^{N}\left(\frac{2}{x_{j}^{2}-x_{k}^{2}}\left(x_{j} \frac{\partial}{\partial x_{j}}-x_{k} \frac{\partial}{\partial x_{k}}\right)\right. \\
& \left.+\frac{K_{j k}-1}{\left(x_{j}-x_{k}\right)^{2}}+\frac{t_{j} t_{k} K_{j k}-1}{\left(x_{j}+x_{k}\right)^{2}}\right) .
\end{aligned}
$$

Since the Hamiltonian is transformed to the Euler operator by

$$
\mathrm{e}^{\frac{1}{4 \omega} \mathcal{O}_{\mathrm{L}}^{B_{N}}}\left(\omega \mathcal{O}_{\mathrm{E}}-\frac{1}{2} \mathcal{O}_{\mathrm{L}}^{B_{N}}\right) \mathrm{e}^{-\frac{1}{4 \omega} \mathcal{O}_{\mathrm{L}}^{B_{N}}}=\omega \mathcal{O}_{\mathrm{E}}
$$

the polynomial part of the non-symmetric orthogonal basis for the $B_{N}$-Calogero Hamiltonian $\hat{H}_{B_{N}}$ is given by

$$
Y_{\lambda_{\sigma}}(\boldsymbol{x} ; 1 / a, 1 / b, \omega)=\mathrm{e}^{-\frac{1}{4 \omega} \mathcal{O}_{\mathrm{L}}^{B_{N}}} \boldsymbol{x}^{\lambda_{\sigma}}
$$


in a similar way to Eq. (四). They are the simultaneous eigenfunctions of the operators $x_{j} \frac{\partial}{\partial x_{j}}$. Also in a similar way to the Calogero model, the non-symmetric orthogonal basis is shown to be different from the known orthogonal basis, namely, the non-symmetric generalized Laguerre polynomials 16,25]. And the number operators are algebraically inequivalent to the known commuting conserved operators constructed by Dunkl operator formulation.

So far, we have not found a similar transformation of the Sutherland model into a decoupled system. This seems rather strange at first because the commuting conserved operators and the Dunkl operators for the Calogero and Sutherland models are known to share the same algebraic structure, and become exactly the same in the limit, $\omega \rightarrow \infty$, (strictly speaking, we have to remove the action to the ground state when we consider the correspondence). The difference of the two models is the structure of the Hamiltonian. While the Calogero Hamiltonian is the simplest conserved operator $\hat{I}_{1}$, the Sutherland Hamiltonian corresponds to the second conserved operator $\hat{I}_{2}$. We have proved that the second conserved operator $\hat{I}_{2}$ can not be constructed from the number operators $\hat{n}_{j}^{\mathrm{C}}$. We think that the point causes the essential difficulty in the application of such a similarity transformation method to the Sutherland model.

Let us summarize this letter. We have shown an algebraic construction of the nonsymmetric orthogonal basis for the Calogero model in completely parallel way to that of the quantum harmonic oscillators. The basis consists of the simultaneous eigenfunctions of the commuting number operators, which is different from the known orthogonal basis spanned by the non-symmetric Hi-Jack polynomials. The commuting number operators are algebraically inequivalent to the known commuting conserved operators given by the Dunkl operator formulation. Existence of the two different sets of commuting conserved operators and the two different orthogonal bases implies some hidden dynamical symmetry behind the model. We have also shown that the same story holds for the $B_{N^{-}}$Calogero model. We have discussed on the difficulty in the application of this similarity-transformation method to the Sutherland model. We hope that the new non-symmetric orthogonal bases will shed new 
light on the study of the eigenfunctions and the correlation functions of the models with or without spins.

The authors appreciate encouraging communications with K. Sogo and F. Göhmann. One of the authors (HU) appreciates the Research Fellowships of the Japan Society for the Promotion of Science for Young Scientists. 


\section{REFERENCES}

* $\quad$ Electronic address: ujino@monet.phys.s.u-tokyo.ac.jp

[1] B. Sutherland, Phys. Rev. A 4, 2019 (1971).

[2] H. Jack, Proc. Roy. Soc. Edinburgh Sect. A 69, 1 (1970).

[3] R. P. Stanley, Adv. Math. 77, 76 (1989).

[4] I. G. Macdonald, Symmetric Functions and Hall Polynomials (Clarendon Press, Oxford, 1995) 2nd ed.

[5] Z. N. C. Ha, Phys. Rev. Lett. 73, 1574 (1994).

[6] F. Calogero, J. Math. Phys. 12, 419 (1971).

[7] H. Ujino and M. Wadati, J. Phys. Soc. Jpn. 63, 3585 (1994).

[8] H. Ujino and M. Wadati, J. Phys. Soc. Jpn. 65, 653 (1996).

[9] H. Ujino and M. Wadati, J. Phys. Soc. Jpn. 65, 2423 (1996).

[10] H. Ujino and M. Wadati, J. Phys. Soc. Jpn. 66, 345 (1997).

[11] C. F. Dunkl, Trans. Am. Math. Soc. 311, 167 (1989).

[12] A. P. Polychronakos, Phys. Rev. Lett. 69, 703 (1992).

[13] K. Sogo, J. Phys. Soc. Jpn. 65, 3097 (1996).

[14] N. Gurappa and P. K. Panigrahi, Report No. cond-mat/9710035.

[15] H. Ujino and M. Wadati, J. Phys. Soc. Jpn. 67, 1 (1998).

[16] T. H. Baker and P. J. Forrester, Nucl. Phys. B492, 682 (1997).

[17] J. F. van Diejen, Commun. Math. Phys. 188, 467 (1997).

[18] S. Kakei, J. Phys. A 29, L619 (1996). 
[19] K. Takemura, J. Phys. A 30, 6185 (1997).

[20] E. M. Opdam, Acta Math. 175, 75 (1995).

[21] D. Bernard, M. Gaudin, F. D. M. Haldane and V. Pasquier, J. Phys. A 26, 5219 (1993).

[22] L. I. Schiff, Quantum Mechanics (McGrawhill Book Company, New York, 1968) 3rd ed.

[23] T. Yamamoto, Phys. Lett. A 208, 293 (1995).

[24] N. Gurappa and P. K. Panigrahi, Report No. quant-ph/9710019.

[25] S. Kakei, J. Phys. A 30, L535 (1997). 